\title{
Modafinil modulation of the default mode network
}

\author{
Michael J. Minzenberg • Jong H. Yoon • \\ Cameron S. Carter
}

Received: 22 July 2010 /Accepted: 22 November 2010 /Published online: 14 December 2010

(C) The Author(s) 2010. This article is published with open access at Springerlink.com

\begin{abstract}
Rationale The default mode network (DMN) is a functional network which is implicated in a range of cognitive processes. This network is proposed to consist of hubs located in the ventromedial prefrontal cortex (vmPFC), posterior cingulate/retrosplenial cortex $(\mathrm{PCC} / \mathrm{rSpl})$, and inferior parietal lobule (IPL), with other midline cortical and temporal lobe nodes connected to these hubs. How this network is modulated by neurochemical systems during functional brain activity is not yet understood.

Objectives In the present study, we used the norepinephrine/dopamine transporter inhibitor modafinil to test the hypothesis that this drug modulates the DMN.

Methods Eighteen healthy right-handed adults participated in a double-blind, placebo-controlled study of single oral dose modafinil $200 \mathrm{mg}$. They performed a simple visual sensorimotor task during slow event-related fMRI. Drug effects were interrogated within the DMN defined by taskinduced deactivation (TID) on placebo.

Results There was a trend toward faster reaction time (RT) on modafinil (Cohen's $d=0.38$ ). Brain regions within the DMN which exhibited significant modafinil-induced augmentation of TID included vmPFC, PCC/rSpl, and left IPL.
\end{abstract}

Electronic supplementary material The online version of this article (doi:10.1007/s00213-010-2111-5) contains supplementary material, which is available to authorized users.

M. J. Minzenberg · J. H. Yoon • C. S. Carter Department of Psychiatry, University of California,

Davis School of Medicine,

Sacramento, CA, USA

M. J. Minzenberg $(\bowtie)$

Imaging Research Center, UC-Davis Health System,

4701 X Street,

Sacramento, CA 95817, USA

e-mail: michael.minzenberg@ucdmc.ucdavis.edu
Across subjects, the modafinil effect on TID in the vmPFC was significantly and specifically associated with drug effects on RT speeding.

Conclusions Modafinil augments TID in the DMN to facilitate sensorimotor processing speed, an effect which may be particularly dependent on changes in vmPFC activity. This is consistent with the gain control function of catecholamine systems and may represent an important aspect of the pro-cognitive effects of modafinil.

Keywords Catecholamines - Default mode network . Modafinil · Task-induced deactivation · Gain control . Negative BOLD response

\section{Introduction}

The default mode network (DMN) is a distributed network of functionally connected cortical regions which can be identified in functional neuroimaging paradigms where task demands are associated with decreased activity relative to a "baseline" (Mazoyer et al. 2001). This is typically referred to as "task-induced deactivation" (TID) and identifies a network that is qualitatively similar to that observed in resting state connectivity (reviewed in Buckner et al. 2008). The DMN contains "hubs" in the ventromedial prefrontal cortex (vmPFC), posterior cingulate/retrosplenial cortex $(\mathrm{PCC} / \mathrm{rSpl})$, and bilateral inferior parietal lobule (IPL). These are strongly connected to each other and to all other nodes of the network (Buckner et al. 2008), and there is evidence that the PCC may be the most prominent functional hub in the brain, from a study that integrated resting-state blood oxygen level-dependent (BOLD) imaging data across 19 independent research sites and 979 subjects (Tomasi and Volkow 2010). The 
DMN is generally active in the relative absence of strong or salient environmental stimuli and is deactivated with attention or control-demanding processes (Fox et al. 2005; McKiernan et al. 2003).

There is now considerable convergent evidence indicating that attention and control processes are strongly modulated by subcortical catecholamine systems (see (Arnsten 1997; Aston-Jones and Cohen 2005; GoldmanRakic et al. 2000; Miller and Cohen 2001; Robbins 2005 for reviews). Cortically projecting systems arising in the locus coeruleus (LC) and the ventral tegmental area exert a gating function for cortical ensembles supporting a given cognitive process (Aston-Jones and Cohen 2005). Catecholamine neurotransmission leads to both the relative suppression of background or spontaneous activity and relative enhancement of excitability to task-relevant excitatory inputs. The combined effect is manifest as an increased gain in the input/output relationships for these cortical neurons, which may be critical to their participation in an active cortical ensemble to support a given cognitive process (Aston-Jones and Cohen 2005).

It remains quite unclear, however, whether this physiological process is employed to modulate cortical regions such as those found in the DMN. A few studies have addressed catecholamine modulation of TID in the DMN, with transient dopamine (DA) depletion (Nagano-Saito et al. 2008), apomorphine administration (Nagano-Saito et al. 2009), natural variation in DA transporter (DAT) binding (Tomasi et al. 2009), and variation in the gene coding for catechol-O-methyl transferase (Liu et al. 2010). These findings generally suggest that higher DA transmission is associated with augmented TID in the DMN.

While DA-regulated TID in DMN areas is observed as a function of parametric task demand, it remains unclear whether catecholamine modulation of the DMN is a predictor of actual task performance. Errors and slowed reaction times (RTs) occurring spontaneously during task performance are preceded within trials by transient attenuations in TID in midline DMN areas (Li et al. 2007; Polli et al. 2005; Weissman et al. 2006). This critically suggests that TID in the DMN, particularly midline areas in vmPFC and $\mathrm{PCC} / \mathrm{rSpl}$, may be a determinant of task performance. This has been shown with single doses of nicotine, which deactivates DMN areas such as the vmPFC and PCC to enhance visuospatial performance (Hahn et al. 2007).

With these considerations, we tested the role of catecholamine systems in the modulation of TID in the DMN and the relationship of this modulation to task performance. We identified the DMN via TID during a visual sensorimotor processing task and used a pharmacological intervention to enhance catecholamine neurotransmission. Modafinil is an FDA-approved agent that inhibits the norepinephrine (NE) transporter (NET) and DAT
(Madras et al. 2006; Volkow et al. 2009), leading to increased synaptic levels of NE and DA, with indirect effects on other neurotransmitter systems (reviewed in Minzenberg and Carter 2008). There is also considerable evidence that modafinil can enhance cognitive performance, including in healthy individuals (reviewed in Minzenberg and Carter 2008). These effects appear to be most strongly manifest on tasks that are highly dependent on the prefrontal cortex (e.g., Turner et al. 2003; see review in Minzenberg and Carter 2008). We hypothesized that modafinil would augment TID in the DMN, as an analogous expression of gain control in support of taskrelevant information processing, and that this effect would be associated with improved performance.

\section{Methods}

\section{Subjects/overview of procedures}

The study was conducted at the University of California, Davis Medical Center from the period March 2006 to January 2008. All procedures were approved by the UCD Institutional Review Board, and all subjects provided informed consent for all procedures. Five subjects consented for the study, but were excluded for the following reasons: Two declined further participation prior to any testing procedures, one tested positive for illicit substances prior to testing, one completed one testing day only (this subject's data were not analyzed), and one experienced equipment malfunction. All additional subjects $(n=18)$ completed this study and provided data for the present analysis. Subjects were all recruited from the community and were included if they were aged 18-50; right-handed; free of past or present psychiatric illness as determined by the Structured Clinical Interview for DSM-IV Disorders, non-patient version; lacked significant medical illness, any neurological illness, and a family history of major psychiatric illness by report; and were not taking medications with either psychotropic effects or established drug-drug interactions with modafinil at study. There were ten men and eight women who completed the study, with mean age 33.6 years. All subjects were instructed to maintain their usual quantities and patterns of nicotine and caffeine intake, without changes on or between test days, in order to avoid neural/cognitive effects due to changes in intake from their baseline. All included subjects tested negative for drugs of abuse in the urine on each testing day. Each completed a double-blind, placebo-controlled crossover study of the effects of modafinil (single oral dose $200 \mathrm{mg}$ ) on neural activity measured by fMRI during performance of a sensorimotor task. We chose $200 \mathrm{mg}$ as a dose that was likely to optimize experimental effects with tolerability in a 
single dose to drug-naïve subjects. Randomization of treatment order was performed without stratification with a computer algorithm by a research pharmacist who also packaged active medication and placebo in identicalappearing capsules for administration and was otherwise uninvolved in the study. Ten subjects completed the active drug testing day first and eight subjects completed the placebo day first, with at least 3 days between test days (mean 5.7 \pm 4.1 days) to allow for complete drug washout. All investigators remained blind to treatment order for individual subjects until all data were acquired for that subject and treatment order information was then necessary to sort data for inferential testing. All subjects received the dose in the morning, and echo-planar imaging was initiated at an average elapsed time $3.6 \pm 0.2 \mathrm{~h}$ post-dose, within the time window of reported average peak plasma levels of modafinil (Robertson and Hellriegel 2003). No subjects reported any adverse subjective or physical symptoms during study procedures.

\section{Task paradigm}

The visual sensorimotor processing task was modified from (Handwerker et al. 2004) and is associated with robust positive event-related activation of the visual cortex and primary motor cortex. It was presented using EPrime software. The trial structure was as follows: A contrastreversing checkerboard was presented for $200 \mathrm{~ms}$; after stimulus offset, the stimulus was replaced with the central fixation cross again. The interstimulus interval was randomly varied between 18 and $22 \mathrm{~s}$ (at 2-s intervals), both to optimize the convolution of the hemodynamic response function (HRF) with the BOLD signal time series and to minimize preparatory responses by subjects. Therefore, the duration of each trial was $18-22 \mathrm{~s}$, and the number of brain volumes acquired on each trial varied from 9 to 11 . Subjects were instructed prior to the task onset to maintain fixation on the crosshair, and then with checkerboard onset, to press a button with the right index finger as fast as possible. One block of 20 trials was presented. To our knowledge, this particular paradigm has not been evaluated to date for TID or other DMN effects.

\section{fMRI acquisition and pre-processing}

Event-related fMRI was conducted on a 3-T Siemens Trio MRI system with a Siemens eight-channel phased array coil. Measurement of BOLD contrast was conducted during single-shot, echo-planar imaging (EPI) using a T2*-weighted sequence and whole brain coverage. The parameters of the EPI sequence were TR 2,000 ms, TE $30 \mathrm{~ms}$, flip angle $90^{\circ}$, FOV $220 \times 220 \mathrm{~mm}$, with 36 contiguous slices in the axial oblique plane with voxel size $3.4 \mathrm{~mm}$ isotropic. Pre- processing was performed using SPM5. The first five images (preceding onset of trial 1 of block 1) were discarded to allow for stabilization of the scanner signal. The remaining images were realigned (motion-corrected) to the first retained image in the first block, then adjusted for acquisition time (slice timing correction), then subject to spatial normalization directly to the EPI template from the SPM5 library. Images were then resliced to $2 \times 2 \times 2$ and spatially smoothed with an 8 -mm, full-width-at-half-maximum Gaussian kernel. At this point, drug and placebo day scans were concatenated for the purpose of modeling of the signal and inferential testing.

Inferential testing of voxel-wise drug effects on neural activity

Derivation of the signal proceeded with the use of the general linear model. Regressors were established for Drug_Event and Placebo_Event. A canonical (doubleGaussian) hemodynamic response function was convolved with a series of delta functions to model the BOLD time series, with regressors placed at event onset. We also established the temporal derivative of the HRF as a regressor, paired with each of the experimental condition regressors, to account for temporal variation in the latency of the event-related response. A $0.0125-\mathrm{Hz}$ high-pass filter was used and a first-order autoregressive function to account for serial autocorrelations. We evaluated the data with global normalization both included and excluded in the model, in parallel analyses. We did this in order to evaluate the potential for detecting spurious deactivations in these data, which have been found by others in BOLD time series data using global normalization (Aguirre et al. 1998; Desjardins et al. 2001; Gavrilescu et al. 2002). A recent paper has found that this effect is minimal in the DMN and that global normalization significantly improves the detection of activity in functional networks that are well established from both animal models and structural anatomic imaging methods in humans (Fox et al. 2009). In the present context, global normalization facilitates the integration of BOLD data across tests days, primarily by accounting for machine-related variation in signal across days. Nevertheless, we evaluated the potential effect of global normalization on the inferential test results by repeating the regression model estimation with the noscaling option in SPM (as per Desjardins et al. 2001). This analysis revealed test statistics virtually identical to that derived with the proportional scaling option, suggesting that this step in the estimation of the regression model does not show spurious effects on inferential tests in this data set. The results of the no-scaling analysis are reported in "Results" below. After signal estimation, linear contrasts were defined at the single-subject level (see below), and 
then relevant contrast maps from individual subjects (containing voxel-wise parameter estimates for a given contrast) were entered into the group-level analysis for inferential testing.

In order to test hypotheses regarding drug effects within the DMN, we interrogated voxels solely within DMN hubs (identified in Buckner et al. 2008). These voxels were identified by meeting each of the following criteria: (a) significant TID in the group-level Placebo_Event minus Baseline contrast at a voxel-wise threshold of $p<0.0005$ uncorrected (negative activations observed in ESM Fig. 1); (b) significant TID in the group-level Drug_Event minus Baseline contrast, also at a voxel-wise threshold of $p<.0005$ uncorrected; (c) located within any of the four following anatomic masks from the AAL library (utilized in the MarsBaR program): bilateral vmPFC (identified in the AAL library as anterior cingulate cortex, which spans the rostral/subgenual ACC and adjacent ventral aspect of the medial frontal gyrus, to comprise the vmPFC), bilateral $\mathrm{rSpl} / \mathrm{PCC}$ (identified as posterior cingulate cortex), and left and right inferior parietal lobules (identified as such; Tzourio-Mazoyer et al. 2002). These conjoined masks (each of which were fixed across subjects) had volumes as follows: vmPFC, 8,072 $\mathrm{mm}^{3} ; \mathrm{rSpl} / \mathrm{PCC}, 2,160 \mathrm{~mm}^{3}$, left IPL, $272 \mathrm{~mm}^{3}$ (see Fig. 1). The right IPL did not contain any voxels that met these criteria, and therefore, drug effects in this region were not interrogated. The use of this DMNdefining procedure is unbiased for testing drug effects and also allows for unambiguous inferences about the direction of these hypothesized treatment effects. This is because any Drug minus Placebo effects manifest as negative values must represent activity further below baseline since activity in these voxels on Placebo is below baseline to start with (i.e., represents TID). For the contrast of Drug_Event minus Placebo_Event, the threshold for statistical significance was set at $p<0.05$, with a smallvolume correction (using the volumes defined by the above criteria) for multiple comparisons. We hypothesized that significant drug effects would be manifest as further deactivations within the $\mathrm{DMN}$, i.e., negative activity in the contrast (Drug_Event minus Placebo_Event).

\section{Region of interest analyses}

We also derived the mean beta values within each of the three aforementioned ROIs (Fig. 1), in each treatment condition, in order to provide a convergent, omnibus test (by ANOVA) of drug effects on TID in these DMN hubs. We also tested the hypothesis that drug effects on TID within these DMN hubs predict drug effects on task performance. For this second test, we established a regression model with $\mathrm{RT}$ change on drug as the dependent variable and stepwise entry of beta change on drug within each of these three ROIs as predictor variables. This analysis allowed us to evaluate the comparative strength with which drug effects in these ROIs predicted performance.

\section{Results}

Effect of modafinil on task performance

There was a trend toward faster RT on drug versus placebo: $421 \pm 130$ versus $476 \pm 158$ ms (Cohen's $d=.38, t=1.46, p=$ 0.08 by one-tailed paired $t$ test).

\section{Effect of modafinil on TID within the DMN}

ESM Fig. 1 depicts the task-related changes in brain activity, with significant deactivation in characteristic DMN regions in response to task demands (i.e., TID). These results are used to define the DMN for interrogation of drug effects. As observed in ESM Fig. 2, drug administration was associated with robust effects in augmentation of TID, within each of the three hubs of the DMN: vmPFC, PCC/rSpl, and left IPL. Clusters within each of these cortical regions exceeded the corrected threshold for statistical significance. Statistics for these clusters are listed in Table 1.

We also defined the task-positive network of activation as voxels with increased activity in the contrast (Placebo_Event minus Baseline) at $p<0.0005$ uncorrected (ESM Fig. 1) and then used this mask to interrogate drug effects on task-positive activity in the contrast (Drug_Event minus Placebo_Event). This analysis revealed no brain regions with changes on drug that met statistical significance (data not shown).

\section{ROI analysis of drug effects on TID}

ANOVA of mean beta values within the ROIs revealed main effects of treatment $(F=7.91, d f=1,17 ; p=0.012)$, ROI $(F=4.00, d f=1,17 ; p=0.039)$, but no significant effects of the treatment-by-ROI interaction $(F=0.17, d f=2,16 ; p=$ 0.845 ). Post hoc analyses of individual ROIs revealed a significant drug effect on betas in each ROI by paired $t$ test (vmPFC, $t=-2.28, p=0.036 ; \mathrm{PCC} / \mathrm{rSpl}, t=-2.59, p=0.019$; left IPL, $t=-2.32, p=0.033$; see Fig. 2, top panel).

Relationship of TID in the DMN hubs to RT

Drug effects on TID in the vmPFC significantly predicted drug effects on RT speeding (adjusted $R^{2}=.26, \beta=.51, F=$ 2.34, $p=.032$; see Fig. 2). After entry of this predictor variable to the regression model, drug effects on TID in the 


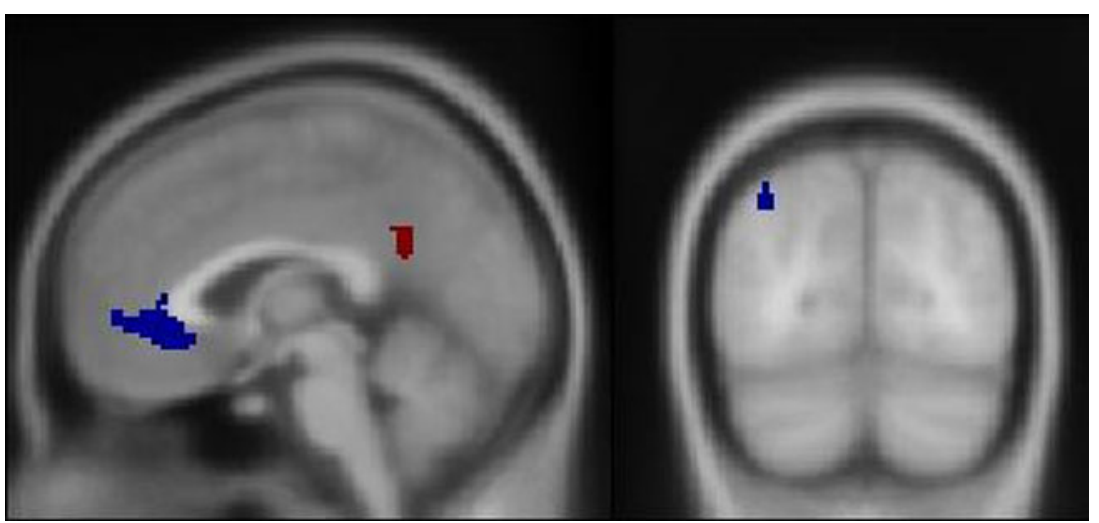

Fig. 1 Brain regions exhibiting task-induced deactivation within the default mode network in both treatment conditions. Standard brain template image depicting the volumes used to interrogate drug effects

other two hubs did not contribute to significant variance in RT speeding across subjects: $\mathrm{rSpl} / \mathrm{PCC}(t=0.78, p=0.45)$ and left IPL $(t=1.5, p=0.16)$. In other words, stronger TID on drug in the vmPFC specifically predicted RT speeding on drug. The linear relationship of drug effects on TID in the vmPFC to drug effects on performance is shown in Fig. 2 (bottom panel).

\section{Discussion}

In this study, we found that a pharmacological intervention which enhances catecholamine neurotransmission augmented the deactivation in the DMN in response to a sensorimotor processing demand. Modafinil, a NET/DAT inhibitor, significantly augmented deactivation in the three major hubs of the DMN - the vmPFC, PCC/rSpl, and IPL. Among these areas, TID in the vmPFC significantly predicted speeding of RT on drug.

There is now considerable evidence that modafinil shows efficacy for a range of cognitive processes, particularly those that are dependent on the PFC (Minzenberg and Carter 2008; Turner et al. 2003). This has been on TID. The vmPFC and PCC/rSpl are shown in the midsagittal plane (left panel) and left IPL shown in the coronal plane (right panel)

demonstrated in healthy populations, sleep-deprived subjects, and a range of neuropsychiatric populations. One very characteristic finding in this literature is that modafinil enhances processing speed, just as it did in the present study. The present evidence therefore suggests that the modulatory effects of modafinil on catecholamine systems may lead to enhanced cognition, in part by augmentation of TID. Additionally, there is evidence that modafinil affects levels of other extracellular neurotransmitters such as serotonin and amino acid neurotransmitters; however, there is no evidence that this agent binds directly to elements of these other neurotransmitter systems, suggesting that these latter effects are mediated via direct catecholamine effects (Minzenberg and Carter 2008). It does remain possible that the drug effects observed here result from indirect modafinil effects on these other neurochemical systems. This seems less likely, however, given (a) the primary effects of this drug on DAT and NET; (b) the literature on modafinil effects on cognition and behavior in animals, which strongly emphasizes catecholamine effects (reviewed in Minzenberg and Carter 2008); (c) our prior evidence of the specific effects of modafinil on task-independent activity in the locus coeruleus using similar fMRI methods (Minzenberg et al. 2008); (d)

Table 1 Brain regions with significant augmentation of task-induced deactivation within the default mode network on modafinil

\begin{tabular}{lcccc}
\hline Brain region & Brodmann area & Volume $\left(\mathrm{mm}^{3}\right)$ & Peak $T$ value & Peak MNI coordinates: $x, y, z$ \\
\hline $\begin{array}{l}\text { Medial frontal gyrus } \\
\text { Left posterior }\end{array}$ & 10 & 5,544 & 4.46 & 0 \\
$\begin{array}{l}\text { Cingulate gyrus } \\
\text { Right posterior }\end{array}$ & $31 / 23$ & 1,512 & 3.68 & -2 \\
$\begin{array}{l}\text { Cingulate gyrus } \\
\text { Left inferior parietal }\end{array}$ & $7 / 31$ & & 3.68 & -4 \\
Lobule & $7 / 19$ & 256 & 28 & -58 \\
\hline
\end{tabular}

Location of clusters with significant drug-related augmentation of TID within the DMN, significant at $p<0.05$ with small-volume correction. See text for details of data acquisition/analysis 

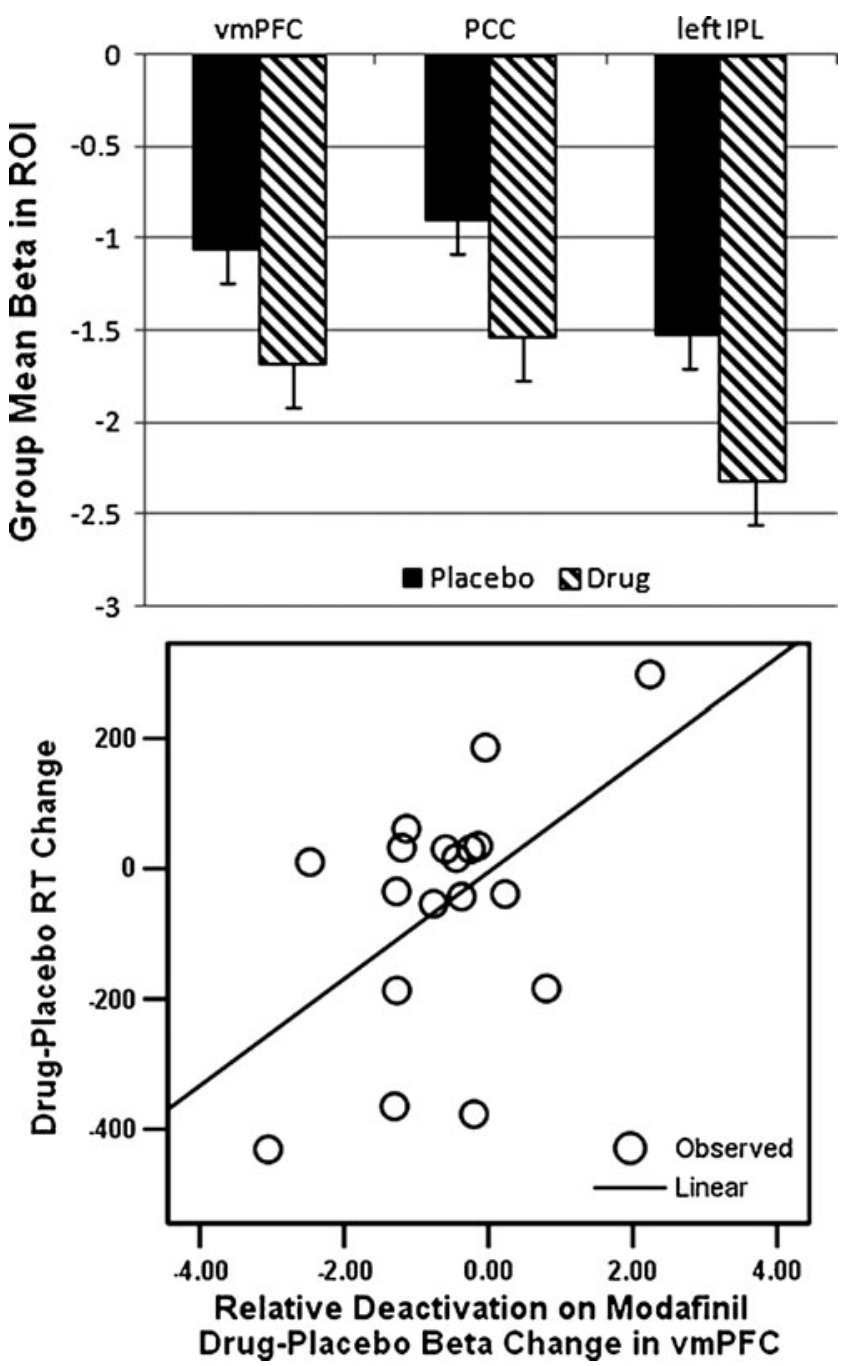

Fig. 2 Modafinil effects on task-induced deactivation in default mode network hubs and association of vmPFC with modafinil effects on task performance. Top panel Mean beta $( \pm \mathrm{SEM})$ within DMN hubs for the drug and placebo effects on TID. Bottom panel Linear correlation between relative deactivation in vmPFC on drug versus placebo (change in mean beta) and relative reaction time on drug versus placebo (change in mean RT). Pearson's $r=0.51, p=0.016$ (one-tailed)

the topographic specificity of the observed effects (e.g., no diffuse effects on positive activity throughout the brain, as might be expected with known modafinil effects observed in animals to increase glutamate and decrease GABA signaling); and (e) the consistency of the present findings with the general model of catecholamine-mediated gain control during information processing (Aston-Jones and Cohen 2005), which emphasizes the modulation of task-relevant brain activity. This notion can be tested more fully in the future by testing the cognitive effects of modafinil in combination with a second catecholamine agent.

Drug-induced modulation of activity in DMN areas was associated with task performance despite the simple sensory and motor demands of the task. Primary sensory and motor areas of the cortex do not participate in the DMN whose hubs generally are not strongly connected to these sensorimotor areas (see Figs. 5-8 in Buckner et al. 2008). This is particularly the case for vmPFC which showed the strongest experimental effects. Nevertheless, TID in vmPFC was a predictor of task performance across subjects. These results indicate that catecholamine systems modulate the DMN and suggest that one informationprocessing role of these systems is to diminish DMN activity in response to task demands. The role of catecholamine systems in cognitive processes such as attention, working and episodic memory, and cognitive control is well established and appears to be expressed via a gating or gain control function in active cortical neuronal ensembles (Aston-Jones and Cohen 2005; Montague et al. 2004). We previously found a complex pattern of effects of modafinil on a rostrodorsal pontine area consistent with the LC, in concert with changes in the cortical-subcortical cognitive control network, during cognitive control task performance (Minzenberg et al. 2008). These effects were consistent with the shift of the LC-NE system to a relatively lowtonic/high-phasic mode of activity, which optimizes taskrelevant activity in cortical ensembles by a gain control mechanism (Aston-Jones and Cohen 2005). The present findings suggest that just as catecholamine neurotransmission augments task-related excitatory throughput in active ensembles, it similarly augments task-related deactivation, and this contributes to task performance.

The association of enhanced TID with enhanced performance may appear paradoxical, given that TID scales with attention demand (McKiernan et al. 2003), which itself tends to lead to decrements in performance, either in accuracy and/or speed. TID can reasonably be considered one important process engaged to meet this demand and support ongoing performance, as a mirror (i.e., "anticorrelated") response to task-positive activity in the frontoparietal network. This perspective is supported by the finding that errors are preceded by transient attenuations in TID in the DMN (Li et al. 2007; Polli et al. 2005; Weissman et al. 2006). Therefore, TID is unlikely to directly lead to performance impairments. This is analogous to the notion that dorsal anterior cingulate activity is associated with errors, but does not cause them, and in fact is engaged in order to positively meet cognitive control demand (van Veen and Carter 2006). This line of reasoning would suggest that a pharmacological intervention that enhances TID should enhance performance in concert. This is the prediction that we have tested with the regression analysis, and the results support this prediction, particularly for the vmPFC. This is a novel finding and, we believe, not inconsistent with the foregoing evidence.

These results also implicate the vmPFC as a particularly strong anatomic substrate for catecholamine effects on TID, 
especially as a determinant of cognitive performance. This cortical area receives significant DA and NE innervation arising from the ventral tegmental area and LC, respectively (reviewed in Steketee 2003). The vmPFC and adjacent orbitofrontal cortex in humans forms a "medial PFC network" projecting to limbic forebrain, hypothalamus, and brainstem areas such as periaqueductal gray, in contrast to the "orbital PFC network" (Ongur and Price 2000). This pattern of connectivity, which likely influences the expression of basic drives, suggests that default mode activity in this cortical area represents the operation of an "intrinsic, probabilistic model of anticipated events" (Raichle and Gusnard 2005) which is altered with the demands of an externally driven, goal-directed task. The distinct patterns of medial PFC network connectivity with the subregions of both the striatum and the mediodorsal nucleus of the thalamus suggest that the medial PFC network may also participate in a unique striato-pallido-thalamo-cortical circuit (Ongur and Price 2000), which could mediate the relationship of TID in vmPFC to task-related neural network activity that serves as a proximal driver of goaldirected task performance. Among the hubs of the DMN, there is evidence that vmPFC and PCC have differing patterns of effective connectivity with their respective anticorrelated dorsal/lateral cortical networks (Uddin et al. 2009) and differing patterns of local functional connectivity density (Tomasi and Volkow 2010). These observations suggest that modulation of vmPFC may have unique consequences (within the DMN) for cortical areas that are brought online during task performance.

The cellular or local network basis of TID, and more generally of BOLD signal changes below baseline, remains to be characterized. However, recent evidence suggests that deactivations, or negative BOLD responses (which can be defined objectively as local increases in oxygen extraction fraction), are associated with increases in local neuronal inhibitory processes, including neuronal membrane hyperpolarization (Boorman et al. 2010; Devor et al. 2007; Northoff et al. 2007; Shmuel et al. 2002). Importantly, this phenomenon may be one mechanism of gain modulation (Wade and Rowland 2010). Hyperpolarization of cortical neuron membranes may be mediated by either gammaamino butyric acid (GABA) receptors or by several monoamine receptor subtypes. Catecholamine systems are well suited to modulate complex local network dynamics that are inhibition-dependent as catecholamine receptors located on both GABAergic interneurons and primary cortical neurons may afford a sensitive control over spatiotemporal patterns of membrane polarization states (Shmuel et al. 2002; Gu 2002). Many important effects of catecholamines on cortical function are mediated via GABAergic interneurons (Bacci et al. 2005; Durstewitz et al. 2000; O'Donnell 2003), and these may be expressed in cortical oscillations such as gamma-range activity. Cortical oscillations such as those in the gamma range are not only associated with BOLD signal change in animal models (Logothetis et al. 2001; Mukamel et al. 2005; Niessing et al. 2005) but are also proposed to represent one important mechanism of gain control in the brain (Salinas and Sejnowski 2001). There is intriguing preliminary evidence that during waking periods, gamma-range power is associated with resting-state fluctuations in BOLD activity (He et al. 2008). It remains unknown whether TID measured in humans during BOLD-fMRI is associated with taskinduced changes in oscillatory activity. It is also important to note that these cortical areas are subject to modulation by other ascending subcortical neurotransmitter systems, and there is evidence, for instance, that nicotinic receptors can mediate deactivation in DMN areas to support cognitive performance (Hahn et al. 2007).

\section{Study limitations}

In the present study, we used a rather simple task with minimal frontoparietal task demands in order to evaluate drug effects on TID in the DMN. The apparent inverse functional relationship between the dorsal/lateral frontoparietal control network and the DMN, which has been reported in other studies (e.g., Fox et al. 2005), is likely an important feature of distributed network activity as it determines cognitive performance. This could even represent a general, or fundamental, feature of cognition as it is instantiated in brain dynamics. With the present experimental design, we are not able to evaluate intra- or internetwork dynamics directly. Nevertheless, we are able to gain a degree of inferential leverage from the lack of engagement of the dorsal frontoparietal network. The present results suggest that catecholamine modulation of DMN in the service of sensorimotor processing is not simply an indirect consequence of the positive modulation of activity in other more dorsal/lateral PFC subregions and the cognitive processes that they subserve. Regarding the question of how neurochemical systems may aid the integration of frontoparietal and DMN networks, a more conclusive answer will require the use of cognitive tasks where these networks can be interrogated concurrently. Given the nearly ubiquitous presence of catecholamines throughout the cortex of humans (Gu 2002), these neurochemical systems seem well suited to integrate the function of these two large, widely distributed cortical networks. Current models of catecholamine-mediated gain control in cortical neuronal ensembles would predict that this anticorrelation should be strengthened with interventions that enhance catecholamine neurotransmission. We also did not conduct other tests of cognition in this study, so the cognitive process specificity of these findings is uncertain. Our working model 
would suggest that augmentation of task-relevant neural changes is a general feature of catecholamine drug effects, but this remains to be fully tested in future work, along with the possible modulation of connectivity within the DMN.

Acknowledgments This work was supported by MH059883 to CSC. This publication was also made possible by grant no. UL1 RR024146 from the National Center for Research Resources (NCRR), a component of the National Institutes of Health $(\mathrm{NIH})$, and NIH Roadmap for Medical Research. Its contents are solely the responsibility of the authors and do not necessarily represent the official view of NCRR or NIH. Information on NCRR is available at http://www. ncrr.nih.gov/. Information on Re-engineering the Clinical Research Enterprise can be obtained from http://nihroadmap.nih.gov/clinical research/overview-translational.asp.

Conflict of interest The authors have no disclosures to make.

Open Access This article is distributed under the terms of the Creative Commons Attribution Noncommercial License which permits any noncommercial use, distribution, and reproduction in any medium, provided the original author(s) and source are credited.

\section{References}

Aguirre GK, Zarahn E, D'Esposito M (1998) The inferential impact of global signal covariates in functional neuroimaging analyses. Neuroimage 8:302-306

Arnsten AF (1997) Catecholamine regulation of the prefrontal cortex. J Psychopharmacol 11:151-162

Aston-Jones G, Cohen JD (2005) An integrative theory of locus coeruleus-norepinephrine function: adaptive gain and optimal performance. Annu Rev Neurosci 28:403-450

Bacci A, Huguenard JR, Prince DA (2005) Modulation of neocortical interneurons: extrinsic influences and exercises in self-control. Trends Neurosci 28:602-610

Boorman L, Kennerley AJ, Johnston D, Jones M, Zheng Y, Redgrave P, Berwick J (2010) Negative blood oxygen level dependence in the rat: a model for investigating the role of suppression in neurovascular coupling. J Neurosci 30:4285-4294

Buckner RL, Andrews-Hanna JR, Schacter DL (2008) The brain's default network: anatomy, function, and relevance to disease. Ann NY Acad Sci 1124:1-38

Desjardins AE, Kiehl KA, Liddle PF (2001) Removal of confounding effects of global signal in functional MRI analyses. NeuroImage 13:751-758

Devor A, Tian P, Nishimura N, Teng IC, Hillman EM, Narayanan SN, Ulbert I, Boas DA, Kleinfeld D, Dale AM (2007) Suppressed neuronal activity and concurrent arteriolar vasoconstriction may explain negative blood oxygenation level-dependent signal. J Neurosci 27:4452-4459

Durstewitz D, Seamans JK, Sejnowski TJ (2000) Dopamine-mediated stabilization of delay-period activity in a network model of prefrontal cortex. J Neurophysiol 83:1733-1750

Fox MD, Snyder AZ, Vincent JL, Corbetta M, Van Essen DC, Raichle ME (2005) The human brain is intrinsically organized into dynamic, anticorrelated functional networks. Proc Natl Acad Sci USA 102:9673-9678

Fox MD, Zhang D, Snyder AZ, Raichle ME (2009) The global signal and observed anticorrelated resting state brain networks. J Neurophysiol 101:3270-3283
Gavrilescu M, Shaw ME, Stuart GW, Eckersley P, Svalbe ID, Egan GF (2002) Simulation of the effects of global normalization procedures in functional MRI. Neuroimage 17:532-542

Goldman-Rakic PS, Muly EC 3rd, Williams GV (2000) D(1) receptors in prefrontal cells and circuits. Brain Res Brain Res Rev 31:295-301

$\mathrm{Gu}$ Q (2002) Neuromodulatory transmitter systems in the cortex and their role in cortical plasticity. Neuroscience 111:815-835

Hahn B, Ross TJ, Yang Y, Kim I, Huestis MA, Stein EA (2007) Nicotine enhances visuospatial attention by deactivating areas of the resting brain default network. J Neurosci 27:3477-3489

Handwerker DA, Ollinger JM, D'Esposito M (2004) Variation of BOLD hemodynamic responses across subjects and brain regions and their effects on statistical analyses. Neuroimage 21:1639-1651

He BJ, Snyder AZ, Zempel JM, Smyth MD, Raichle ME (2008) Electrophysiological correlates of the brain's intrinsic large-scale functional architecture. Proc Natl Acad Sci USA 105:1603916044

Li CS, Yan P, Bergquist KL, Sinha R (2007) Greater activation of the "default" brain regions predicts stop signal errors. Neuroimage 38:640-648

Liu B, Song M, Li J, Liu Y, Li K, Yu C, Jiang T (2010) Prefrontalrelated functional connectivities within the default network are modulated by COMT val ${ }^{158}$ met in healthy young adults. J Neurosci 30:64-69

Logothetis NK, Pauls J, Augath M, Trinath T, Oeltermann A (2001) Neurophysiological investigation of the basis of the fMRI signal. Nature 412:150-157

Madras BK, Xie Z, Lin Z, Jassen A, Panas H, Lynch L, Johnson R, Livni E, Spencer TJ, Bonab AA, Miller GM, Fischman AJ (2006) Modafinil occupies dopamine and norepinephrine transporters in vivo and modulates the transporters and trace amine activity in vitro. J Pharmacol Exp Ther 319:561-569

Mazoyer B, Zago L, Mellet E, Bricogne S, Etard O, Houde O, Crivello F, Joliot M, Petit L, Tzourio-Mazoyer N (2001) Cortical networks for working memory and executive functions sustain the conscious resting state in man. Brain Res Bull 54:287-298

McKiernan KA, Kaufman JN, Kucera-Thompson J, Binder JR (2003) A parametric manipulation of factors affecting task-induced deactivation in functional neuroimaging. $J$ Cogn Neurosci 15:394-408

Miller EK, Cohen JD (2001) An integrative theory of prefrontal cortex function. Annu Rev Neurosci 24:167-202

Minzenberg MJ, Carter CS (2008) Modafinil: a review of neurochemical actions and effects on cognition. Neuropsychopharmacology 33:1477-1502

Minzenberg MJ, Watrous AJ, Yoon JH, Ursu S, Carter CS (2008) Modafinil shifts human locus coeruleus to low-tonic, high-phasic activity during functional MRI. Science 322:1700-1702

Montague PR, Hyman SE, Cohen JD (2004) Computational roles for dopamine in behavioural control. Nature 431:760-767

Mukamel R, Gelbard H, Arieli A, Hasson U, Fried I, Malach R (2005) Coupling between neuronal firing, field potentials, and FMRI in human auditory cortex. Science 309:951-954

Nagano-Saito A, Leyton M, Monchi O, Goldberg YK, He Y, Dagher A (2008) Dopamine depletion impairs frontostriatal functional connectivity during a set-shifting task. J Neurosci 28:3697-3706

Nagano-Saito A, Liu J, Doyon J, Dagher A (2009) Dopamine modulates default mode network deactivation in elderly individuals during the Tower of London task. Neurosci Lett 458:1-5

Niessing J, Ebisch B, Schmidt KE, Niessing M, Singer W, Galuske RA (2005) Hemodynamic signals correlate tightly with synchronized gamma oscillations. Science 309:948-951

Northoff G, Walter M, Schulte RF, Beck J, Dydak U, Henning A, Boeker H, Grimm S, Boesiger P (2007) GABA concentrations in the human anterior cingulate cortex predict negative BOLD responses in fMRI. Nat Neurosci 10:1515-1517 
O’Donnell P (2003) Dopamine gating of forebrain neural ensembles. Eur J Neurosci 17:429-435

Ongur D, Price JL (2000) The organization of networks within the orbital and medial prefrontal cortex of rats, monkeys and humans. Cereb Cortex 10:206-219

Polli FE, Barton JJ, Cain MS, Thakkar KN, Rauch SL, Manoach DS (2005) Rostral and dorsal anterior cingulate cortex make dissociable contributions during antisaccade error commission. Proc Natl Acad Sci USA 102:15700-15705

Raichle ME, Gusnard DA (2005) Intrinsic brain activity sets the stage for expression of motivated behavior. J Comp Neurol 493:167-176

Robbins TW (2005) Chemistry of the mind: neurochemical modulation of prefrontal cortical function. J Comp Neurol 493:140-146

Robertson P Jr, Hellriegel ET (2003) Clinical pharmacokinetic profile of modafinil. Clin Pharmacokinet 42:123-137

Salinas E, Sejnowski TJ (2001) Gain modulation in the central nervous system: where behavior, neurophysiology, and computation meet. Neuroscientist 7:430-440

Shmuel A, Yacoub E, Pfeuffer J, Van de Moortele PF, Adriany G, Hu X, Ugurbil K (2002) Sustained negative BOLD, blood flow and oxygen consumption response and its coupling to the positive response in the human brain. Neuron 36:1195-1210

Steketee JD (2003) Neurotransmitter systems of the medial prefrontal cortex: potential role in sensitization to psychostimulants. Brain Res Brain Res Rev 41:203-228

Tomasi D, Volkow ND (2010) Functional connectivity density mapping. PNAS 107(21):9885-9890

Tomasi D, Volkow ND, Wang R, Telang F, Wang GJ, Chang L, Ernst T, Fowler JS (2009) Dopamine transporters in striatum correlate with deactivation in the default mode network during visuospatial attention. PLoS ONE 4:e6102

Turner DC, Robbins TW, Clark L, Aron AR, Dowson J, Sahakian BJ (2003) Cognitive enhancing effects of modafinil in healthy volunteers. Psychopharmacology (Berl) 165:260-269

Tzourio-Mazoyer N, Landeau B, Papathanassiou D, Crivello F, Etard O, Delcroix N, Mazoyer B, Joliot M (2002) Automated anatomical labeling of activations in SPM using a macroscopic anatomical parcellation of the MNI MRI single-subject brain. Neuroimage 15:273-289

Uddin LQ, Kelly AM, Biswal BB, Xavier Castellanos F, Milham MP (2009) Functional connectivity of default mode network components: correlation, anticorrelation, and causality. Hum Brain Mapp 30:625-637

van Veen V, Carter CS (2006) Error detection, correction, and prevention in the brain: a brief review of data and theories. Clin EEG Neurosci 37:330-335

Volkow ND, Fowler JS, Logan J, Alexoff D, Zhu W, Telang F, Wang GJ, Jayne M, Hooker JM, Wong C, Hubbard B, Carter P, Warner D, King P, Shea C, Xu Y, Muench L, Apelskog-Torres K (2009) Effects of modafinil on dopamine and dopamine transporters in the male human brain: clinical implications. JAMA 301:11481154

Wade AR, Rowland J (2010) Early suppressive mechanisms and the negative blood oxygenation level-dependent response in human visual cortex. J Neurosci 30:5008-5019

Weissman DH, Roberts KC, Visscher KM, Woldorff MG (2006) The neural bases of momentary lapses in attention. Nat Neurosci 9:971-978 